\title{
Groundwater recharge through wells in open loop geothermal system: problems and solutions - part 1
}

\section{Ricarica delle acque sotterranee mediante pozzi in sistemi geotermici a circuito aperto: problemi e soluzioni - parte 1}

Giovanni Pietro Beretta Università di Milano, Dipartimento di Scienze della Terra “Ardito Desio"- via Mangiagalli 34, 20133 Milano

giovanni.beretta@unimi.it

Parole chiave: ciclo aperto, bassa entalpia, pompe di calore geotermiche, pozzi di ricarica, intasamento.

Keywords: open loop, low enthalpy, geothermal heat pump, recharge wells, clogging.

Riassunto: Sono descritti, nelle due parti di cui si compone l'articolo, i problemi connessi alla gestione dei pozzi per acqua finalizzati all'utilizzo di un impianto di geotermia a bassa entalpia mediante pompe di calore.

In molti casi, per assenza di recapiti superficiali o e/o per garantire la conservazione della risorsa anche dal punto di vista quantitativo, viene previsto un doppietto con un pozzo di presa e un pozzo di resa.

Per questa tipologia di installazione viene considerato principalmente il fenomeno dell'intasamento, che interessa pozzi con funzioni differenti e con potenziale termico diverso connessi al prelievo e alla reimmissione di acque nel sottosuolo; questo fenomeno è dovuto a presenza di bolle d'aria, di solidi sospesi, di crescita batterica e a reazioni chimico-fisiche che sono descritte nel testo.

Oltre a riconoscere le modalità di gestione dei pozzi di ricarica per evitare la fratturazione di dreno e cementazione, è suggerita poi la modalità di impostazione delle prove che sono utilizzate per dimensionare correttamente le portate di esercizio dei pozzi (presa-resa) da cui dipende l'efficienza e l'efficacia dell'impianto geotermico e la sua resa economica.Viene infine illustrata una normativa regionale sulla qualità delle acque usate nel ciclo aperto.

Abstract: In the two parts of this article, the problems related to the management of water wells as part of a low-enthalpy geothermal power plant by means of heat pumps (open loop system) are described. In many cases, in absence of discharge in surface water andlor to ensure the conservation of the resource from a quantitative point of view, a doublet system constituted by a pumping and a recharge well is provided. The clogging phenomenon often occurs in this kind of plant, affecting wells with different functions and different thermal potential related to water withdrawal and reinjection into the ground. This phenomenon is due to the presence of air bubbles, suspended solids, bacterial growth and to the chemical-physical reactions that are described in the text. Besides acknowledging the activities for recharge wells management to avoid the fracturing of the drain and the cementation, is also suggested the procedure of in situ tests that are used to properly determine the optimal well discharge, which determines the efficiency and effectiveness of the geothermal system and its economic performance.It is finally shown a regional regulation on the water quality used in an open loop.

\section{Introduzione}

Negli ultimi anni l'accresciuta attenzione ai problemi di gestione e protezione dell'acqua ha comportato un nuovo interesse verso la ricarica artificiale, per il miglioramento degli aspetti quantitativi e qualitativi della risorsa.

La ricarica artificiale delle acque sotterranee negli acquiferi tradizionalmente sfruttati è prevista con idonei manufatti secondo tre finalità, previste anche dalle diverse norme riguardanti le acque:

- Ravvenamento e accrescimento artificiale dei corpi idrici sotterranei ai fini del raggiungimento degli obiettivi di qualità (D.M. n.100/2016). Questa tipologia di intervento appare principalmente finalizzato al raggiungimento dello stato di buono per la qualità in quanto si cita la necessità del contrasto ad "una tendenza significativa e duratura all'aumento delle concentrazioni di inquinanti". Esperienze in merito a vasche disperdenti di acque nel sottosuolo sono state realizzate in Lombardia, Emilia-Romagna e Veneto.

- Messa in sicurezza di siti contaminati in presenza di barriere idrauliche. Questa tipologia di ricarica, prevista anch'essa dalla Legge, n. 98/2013, viene adottata per poter ristabilire lo stato quantitativo della risorsa nel caso siano attivi pozzi di prelievo di acque contaminate; ciò anche in relazione alla possibile assenza di recapiti locali delle acque emunte dopo il trattamento. Oltre a tale funzione si registra anche l'esigenza di ostacolare l'ingressione di acque salate in zone costiere, laddove si abbia un'area industriale con presenza di barriere idrauliche per la bonifica/messa in sicurezza, come nel caso dei Siti di Interesse Nazionale di Napoli-Bagnoli e di Manfredonia.

- Utilizzo di energia geotermica a bassa entalpia $(<90$ ${ }^{\circ} \mathrm{C}$ ) in sistemi a ciclo aperto. La normativa sulle piccole utilizzazioni locali è costituita dal D.Lgs. 22/2010 e sue modificazioni e integrazioni. Successivamente al prelievo delle acque e allo sfruttamento del loro potenziale termico in pompe di calore, le acque di scarico possono essere destinate a corpi idrici superficiali, a sistemi di collettamento delle acque o direttamente ad acque 
sotterranee. Il D.lgs. 152/06 prevede anche quest'ultima opzione così come la Direttiva 2000/60/UE. In presenza di un diffuso uso delle pompe di calore risulta necessario non depauperare eccessivamente le risorse idriche sotterranee e pertanto la ricarica diventa un'opzione preferibile, anche a fronte dell'esigenza altrettanto importante di garantire un livello piezometrico sostenibile nei confronti del sollevamento della falda conseguente alla riduzione generalizzata dei prelievi idrici, come a Milano. Permane qualche difficoltà autorizzativa nel caso le acque prelevate e da reiniettare non rispettino gli obiettivi di qualità della Direttiva 2006/118/CE e del D.Lgs. 30/2009, seppure non degradate dall'uso nelle pompe di calore, ma presenti in aree con inquinamento diffuso o proveniente da sorgenti poste a monte. Su questi aspetti è stata predisposta una normativa regionale di seguito commentata.

Nella successiva trattazione si approfondisce l'argomento relativo alla reimmissione delle acque nel sottosuolo dopo il loro utilizzo geotermico mediante pompe di calore, analizzando le diverse problematiche connesse alla installazione e alla gestione dei pozzi, escludendo per ragioni di sintesi altre tipologie di installazioni.

Dopo una breve introduzione sulla potenzialità degli impianti e sulla disposizione dei pozzi, viene in particolare esaminato il problema dell'intasamento (clogging) che si verifica soprattutto nei pozzi di ricarica, essendo queste opere progettate e costruite similmente a quelle che sono destinate alla captazione delle acque sotterranee e generalmente non tenendo in conto della diversità dei flussi idrici e delle modifiche alle condizioni fisico-chimiche e biologiche che si introducono nel sottosuolo.

Un ulteriore aspetto trattato riguarda le cautele da adottare per evitare effetti di fratturazione del terreno, particolarmente negativo per la tenuta del dreno e della cementazione del pozzo.

Si conclude con un esempio di una migliore conoscenza dal punto di vista pratico del funzionamento della coppia pozzo di presa-pozzo di resa ai fini di garantire una sua migliore efficienza.

Non sono considerati nel testo, per ragioni di sintesi, gli interventi atti a ripristinare la funzionalità di pozzi intasati.

Allo stesso modo non si considerano le peculiari modalità e problematiche connesse alla reiniezione, nelle stesse formazioni geologiche profonde da cui provengono, delle acque saline di strato, separate dagli idrocarburi, derivanti dall'esplorazione e sfruttamento dei giacimenti di idrocarburi.

\section{Fattibilità idrogeologica e idrochimica del ciclo aperto nelle pompe di calore}

Nell'ambito delle varie richieste energetiche delle zone urbane, che sono indicate in Tab. 1 in funzione dell'uso del suolo e degli insediamenti presenti o da realizzare, si deve valutare la fattibilità di una pompa di calore a circuito aperto mediante la fornitura di acque sotterranee che hanno un potenziale termico pressoché costante.

Nello schema si osserva con evidenza e come intuibile che ad una maggiore richiesta deve corrispondere anche un maggiore utilizzo delle acque, ovviamente a parità di prestazioni delle
Tab. 1 - Typical capacity of different low enthalpy heat pump (Le Feuvre, St John Cox 2009).

Tab. 1 - Capacità richieste per pompe di calore a bassa entalpia (Le Feuvre, St John Cox 2009).

\begin{tabular}{|c|c|c|}
\hline Uso & Capacità & Valori di riferimento \\
\hline Residenziale-Domestico & $2-15 \mathrm{~kW}$ & $\begin{array}{l}\text { Piccolo: } 5 \mathrm{~kW} \\
\text { (case a schiera) } \\
\text { Grande: } 11 \mathrm{~kW} \\
\text { (case indipendenti) }\end{array}$ \\
\hline Residenziale-Collettivo & $20-100 \mathrm{~kW}$ & $\begin{array}{l}\text { Dipende dal numero e } \\
\text { dalla disposizione delle } \\
\text { unità abitative }\end{array}$ \\
\hline Commerciale & $\begin{array}{c}50 \mathrm{~kW}-\mathrm{MW} \\
\text { (scala) }\end{array}$ & $\begin{array}{l}\text { Piccolo: } 55 \mathrm{~kW} \\
\text { (piccoli uffici) } \\
\text { Grande: } 300 \mathrm{~kW} \\
\text { (grandi uffici) }\end{array}$ \\
\hline Edifici pubblici & $\begin{array}{c}50 \mathrm{~kW}-\mathrm{MW} \\
\text { (scala) }\end{array}$ & $\begin{array}{l}\text { Uffici: simile al } \\
\text { commerciale } \\
\text { Scuole: considerato } \\
\text { sulle dimensioni e sui } \\
\text { vari servizi } \\
\text { Ospedali: ordine di } \\
\text { grandezza del MW }\end{array}$ \\
\hline
\end{tabular}

pompe di calore che hanno dal punto di vista tecnologico coefficienti di prestazioni COP differenti (ad es. COP=3).

$\mathrm{Si}$ illustrano preliminarmente alcune condizioni idrogeologiche e idrochimiche a grande scala che possono condizionare la scelta di un sistema a ciclo aperto di una pompa di calore.

Come viene riassunto nella Tab. 2 sono considerati tre parametri che individuano la potenzialità dell'acquifero nel fornire acqua e nei vantaggi dal punto di vista pratico (costi di perforazione ed energetici connessi al sollevamento delle acque) e di impatto ambientale (possibilità di reiniezione delle acque senza loro affioramento o invasione di strutture nel sottosuolo ed eventuale intasamento delle opere).

Tab. 2 - Parameter ranges and weighting coefficients for installation open loop cycle heat pumps (Schomburgk et al. 2005. Copyright (C) BRGM - Rapport RP-53306-FR).

Tab. 2 - Pesi e punteggi relativi all'installazione di pompe di calore a circuito aperto (Schomburgk et al. 2005. Copyright (C) BRGM-Rapport RP-53306-FR).

\begin{tabular}{|l|c|c|c|}
\hline Peso & 1 & 2 & 3 \\
\hline Soggiecenza falda $(\mathrm{m})$ & $0-5$ o $>50$ & $25-50$ & $5-25$ \\
\hline Spessore acquifero $(\mathrm{m})$ & $0-5$ & $5-20$ & $>20$ \\
\hline Trasmissività $\left(\mathrm{m}^{2} / \mathrm{s}\right)$ & $10^{-5}-10^{-3}$ & $1-210^{-3}$ & $>10^{-2}$ \\
\hline Idrochimica: durezza $\left({ }^{\circ} \mathrm{F}\right)$ & $>32$ & $22-32$ & $<22$ \\
\hline
\end{tabular}

Sono riportati anche i pesi attribuiti a tali diversi parametri che consentono poi di fornire un giudizio qualitativo circa la convenienza di una installazione del tipo considerato (Tab. 3)

Di seguito si pone l'attenzione alle condizioni che determinano una possibile riduzione della potenzialità dell'impianto progettato connesso al maggiore inconveniente che si può manifestare nella sua gestione, connesso all'intasamento soprattutto dell'opera di reimmissione delle 
Tab. 3 - Exploitability ratings as inferred from parameter weights (Schomburgk et al. 2005. Copyright (C) BRGM - Rapport RP-53306-FR).

Tab. 3 - Sfruttabilità di pompe di calore a ciclo aperto desunta dai pesi dei parametri (Schomburgk et al. 2005. Copyright (C) BRGM - Rapport RP-53306-FR).

\begin{tabular}{|c|c|c|c|c|c|c|c|c|c|}
\hline Trasmissività & \multicolumn{3}{|c|}{1} & \multicolumn{3}{|c|}{2} & \multicolumn{3}{|c|}{3} \\
\hline Spessore acquifero & 1 & 2 & 3 & 1 & 2 & 3 & 1 & 2 & 3 \\
\hline Soggiacenza falda & 1 & 2 & 3 & 1 & 2 & 3 & 1 & 2 & 3 \\
\hline Idrochimica: durezza & 1 & 2 & 3 & 1 & 2 & 3 & 1 & 2 & 3 \\
\hline Possibile utilizzo & 4 & 7 & 10 & 5 & 8 & 11 & 6 & 9 & 12 \\
\hline Commento sull'utilizzo & Molto debole & Medio & Forte & Debole & Medio & Molto forte & Debole & Forte & Molto forte \\
\hline
\end{tabular}

acque nel sottosuolo, dopo il loro sfruttamento dal punto di vista del potenziale termico.

\section{Disposizione e funzionamento dei pozzi nel ciclo aperto}

Nel ciclo aperto di sfruttamento dell'energia geotermica a bassa entalpia sono previsti sostanzialmente tre tipi di installazioni che interessano le acque sotterranee:

- pozzi a destinazione fissa: viene sempre mantenuta la funzionalità delle opere e si ha un pozzo di sola presa e un pozzo di sola resa;

- $\quad$ pozzi anisotropi: come nel caso precedente, ma i pozzi hanno profondità differenti all'interno dello stesso acquifero;

- pozzi a destinazione alterna: viene modificata stagionalmente la modalità di funzionamento, con il pozzo di presa che diventa di resa e viceversa.

Una prima difficoltà di realizzazione è costituita dall'interferenza termica da evitare o almeno da minimizzare e ad esempio Banks 2009 riferisce che con una trasmissività di circa $10^{-3} \mathrm{~m}^{2} / \mathrm{s}$, un gradiente idraulico di 0.01 e una portata di $5 \mathrm{l} / \mathrm{s}$ si deve mantenere un interasse di circa $275 \mathrm{~m}$ tra i pozzi per non avere interferenza, ma questa distanza è irrealistica in ambiente urbano.

Un calcolo preliminare può essere infatti effettuato nel modo di seguito descritto per un pozzo di presa immediatamente a monte di un pozzo di resa secondo la direzione di flusso idrico e con le consuete approssimazioni relative ai calcoli con modelli analitici (ad esempio: mezzo omogeneo, assenza di ricarica, etc.).

Secondo l'approccio suggerito da Lippmann e Tsang 1980 viene calcolato il parametro adimensionale $\beta$ dato da:

$\beta=\frac{2 Q}{\pi T i d}$

essendo Q la portata $\left(\mathrm{m}^{3} / \mathrm{s}\right)$, T la trasmissività dell'acquifero $\left(\mathrm{m}^{2} / \mathrm{s}\right)$ il gradiente idraulico e d (m) la distanza tra i pozzi.

Il tempo $\mathrm{t}_{\mathrm{a}}$ (s) di apparizione dell'anomalia termica al pozzo di presa è dato dalla seguente relazione, con n porosità efficace e k conducibilità idraulica dell'acquifero $(\mathrm{m} / \mathrm{s})$ :

$$
t_{a}=\frac{n d}{k i}\left[\left(\frac{\beta}{\sqrt{\beta-1}}\right) \tan ^{-1}\left(\frac{1}{\sqrt{\beta-1}}\right)-1\right]
$$

È inoltre possibile stimare anche il tasso di riciclaggio delle acque $\mathrm{R}$ (prelievo dal pozzo di presa dell'acqua iniettata nel limitrofo pozzo di resa con conseguente modifica del potenziale termico) a regime stazionario mediante la relazione:

$$
R=1-\frac{2}{\pi}\left[\tan ^{-1}\left(\frac{1}{\sqrt{\beta-1}}\right)+\left(\frac{\sqrt{\beta-1}}{\beta}\right)\right]
$$

Questo approccio bidimensionale appare semplificato e può produrre un risultato più cautelativo in quanto, considerando la natura tridimensionale del fenomeno, oltre al trasporto di calore per avvezione nell'acquifero si ha anche un trasporto di calore per conduzione negli acquitardi/acquicludi presenti nel sottosuolo in vicinanza dell'acquifero sfruttato e per perdite in atmosfera se si ha una bassa soggiacenza.

Ai fini operativi è interessante l'applicazione dei pozzi anisotropi in zone urbanizzate, in quanto consente una riduzione dell'interasse dei pozzi minimizzando il tasso di riciclaggio, vista la necessità di mantenere le strutture all'interno di una stessa proprietà.

Lo schema di tale installazione prevede, all'interno di uno stesso acquifero, un pozzo di presa più profondo e uno di resa più superficiale e la formazione di un reticolo di flusso illustrato in Fig. 1.

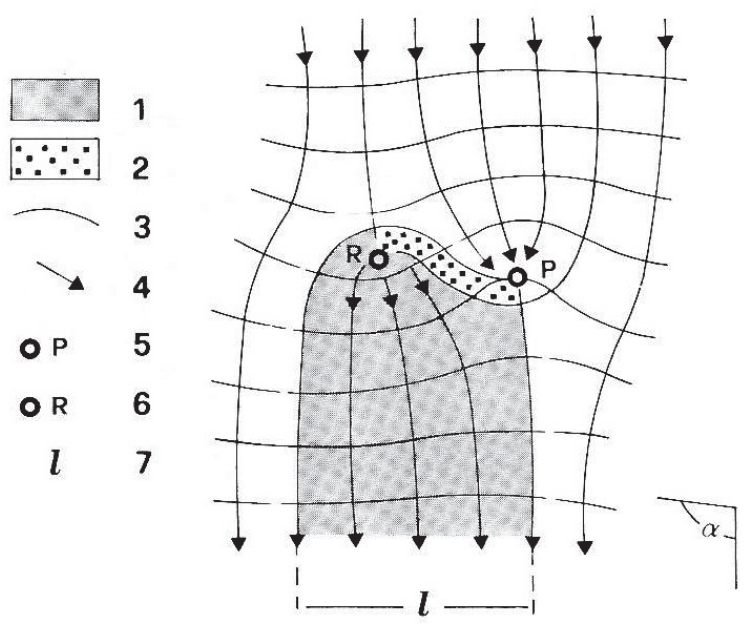

Fig. 1 - Flow net scheme close to couple of anisotropic wells. 1-Recharge well zone (thermal front), 2 -recycling zone, 3 -equipotential line, 4 -pumping well, 5 - recharge well, 7 - thermal front width (Beretta 1984)

Fig. 1 - Schema della deformazione del reticolo di flusso in prossimità di una coppia di pozzi anisotropi. 1- zona interessata dalla ricarica del pozzo (fronte termico), 2-zona di riciclaggio, 3- equipotenziale, 4 - linea di flusso, 5- pozzo di prelievo, 6- pozzo di reiniezione, 7 - larghezza del fronte termico (Beretta 1984) 
In prima approssimazione può essere adottato il seguente schema di dimensionamento (Fig. 2), essendo b lo spessore dell'acquifero $(\mathrm{m}), \mathrm{v}$ la velocità di Darcy $(\mathrm{m} / \mathrm{s})$ e $\alpha\left(^{\circ}\right)$ l'angolo formato dalla congiungente i pozzi di resa e di presa e la direzione del flusso idrico sotterraneo e M:

$$
M=\frac{2 Q}{b d v}
$$

In una successiva fase di approfondimento e soprattutto per installazione di campi pozzi deve essere effettuata una elaborazione con modello numerico.

Si sottolinea che il riciclaggio si registra con maggiore impatto nei pozzi a destinazione fissa, mentre viene modificato in modo significativo nei pozzi a destinazione alterna.

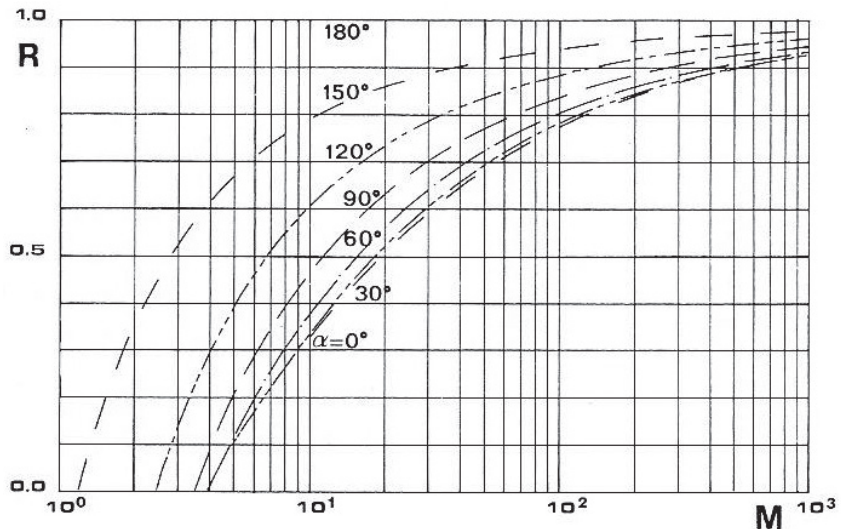

Fig. 2 - Diagram of recycling rate $R$ versus $M$ and $\alpha$ (Ausseur, Sauty 1982 - Permission for publishing from La Houille Blanche-Societé, Hydrotecnique de France).

Fig. 2 - Diagramma per il calcolo del tasso di riciclaggio $\mathrm{R}$ in funzione di $\mathrm{M}$ ed $\alpha$ (Ausseur, Sauty 1982 - Permission for publishing da La Houille BlancheSocieté, Hydrotecnique de France).

\section{Intasamento dei pozzi per acqua per la ricarica artificiale}

Un significativo problema connesso alla gestione dei pozzi per acqua e soprattutto per quelli di ricarica artificiale è dovuto all'intasamento (clogging), che si può verificare durante la vita dell'opera con diversa velocità.

Questo fenomeno, che riduce l'efficienza del pozzo anche in modo sensibile, si sviluppa principalmente in base a due fattori:

- $\quad$ strutturale: i pozzi di resa sono progettati e realizzati con le stesse modalità e strutture che sono utilizzate per i pozzi di presa, pur essendo il flusso idrico controcorrente rispetto a quello previsto per i filtri e il dreno;

- gestionale: l'introduzione di acque nel sottosuolo, seppure derivanti dallo stesso acquifero, possono comunque produrre reazioni chimico-fisiche e biologiche che diminuiscono l'efficienza delle opere.

Lattenzione viene di seguito concentrata su quest'ultimo aspetto $\mathrm{e}$ in particolare si riporta in Fig. 3 uno schema concettuale dell'evoluzione della difficoltà, che si producono nel tempo in un pozzo, all'introduzione di acque in un acquifero definite con il termine di "resistenza al flusso".

Si osserva in ordine di tempo l'importanza dell'intasamento dovuto alle bolle d'aria, seguito dai solidi sospesi (presenti originariamente nell'acqua o formatisi per reazioni di precipitazione o di corrosione delle tubazioni) e dalla crescita batterica.

Si esaminano pertanto in questo ordine i diversi fenomeni precedentemente illustrati che, se si verificano effettivamente, obbligano a costose operazioni di ripristino dell'efficienza dei pozzi, che non sempre possono ripristinare le prestazioni originarie fino alla necessità di dover realizzare nuovi pozzi in sostituzione di quelli obsoleti.

I contenuti delle descrizioni di seguito proposte sono in gran parte ripresi dalla letteratura tecnico-scientifica disponibile sull'argomento che è citata nel testo.

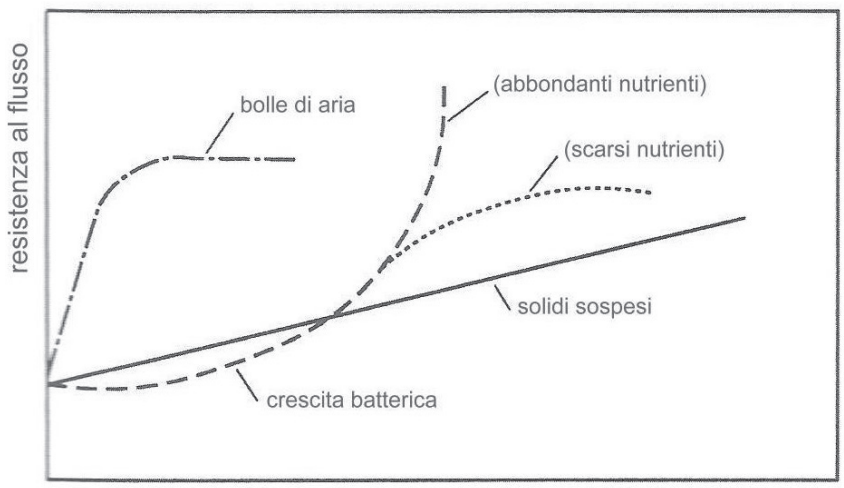

tempo

Fig. 3 - Scheme of factors affecting the groundwater flow resistance in a well (Pyne 2005).

Fig. 3 - Schema dei fattori che determinano la resistenza al flusso delle acque sotterranee in un pozzo (Pyne 2005).

\section{Presenza e formazione di bolle d'aria}

Le bolle d'aria che si possono formare in un pozzo (Fig. 4) hanno un diametro compreso tra 1 e $10 \mathrm{~mm}$ e risalgono luogo l'acqua internamente al tubo ad una velocità $0.3-0.4 \mathrm{~m} / \mathrm{s}$. Nella maggior parte dei pozzi di iniezione possono quindi raggiungere agevolmente $\mathrm{i}$ filtri, penetrare nel dreno e poi nell'acquifero, bloccandone i pori effettivamente disponibili al flusso idrico (porosità efficace) in modo estremamente rapido.

Quando la linea di iniezione termina al fondo del pozzo, le bolle risalendo possono intasare completamente i filtri; se questa linea termina sopra i filtri questo non può accadere, poiché la velocità verticale dell'acqua diminuisce più in profondità e le bolle non saranno in grado di raggiungere la parte più bassa del filtro.

Ridurre la portata in un pozzo con l'aumento dell'intasamento provocherà una stabilizzazione ad un certo livello e si verificherà un equilibrio tra formazione e dissoluzione.

L'intasamento da bolle di gas è caratterizzata dal suddetto equilibrio e la rapidità con cui si potrà intasare il pozzo dopo essere messi in servizio.

$\mathrm{Va}$ anche considerato che in presenza di una elevata soggiacenza della falda (ad esempio oltre 20-25 m) si crea un consistente serbatoio di aria che sovrasta il mezzo saturo e, nel caso di una rapida manovra che determina una risalita 
veloce delle acque in un pozzo di ricarica, si possono creare le condizioni per inglobare bolle di gas che generano i problemi sopra accennati. In ogni caso i filtri del pozzo non devono essere esposti al mezzo insaturo durante la gestione dell'impianto.

Per quanto riguarda la natura dei gas disciolti, concentrando l'attenzione sulle falde superficiali si osserva che:

- Il contenuto di $\mathrm{N}_{2}$ è simile a quello atmosferico;

- Il contenuto di $\mathrm{O}_{2}$ è inferiore a quello atmosferico in seguito alle reazioni con minerali e sostanza organica nel sottosuolo;

- la pressione parziale della $\mathrm{CO}_{2}$ è maggiore di quella atmosferica in seguito alla respirazione di batteri del suolo, decomposizione di sostanza organica e reazioni di acidi nei carbonati;

- altri gas come $\mathrm{CH}_{4}$ possono essere presenti in casi subordinati, come in presenza di acque metanigene di origine naturale (bassa Pianura Padana) o di reazioni di trasformazione di composti organici naturali o di produzione antropica.

Gli impianti di degassaggio delle acque prima della loro reimmissione sono poco diffusi sia per il costo sia perché il degassaggio ad esempio della $\mathrm{CO}_{2}$ può determinare la precipitazione della calcite, incrementando il problema dell'intasamento del pozzo.

Diverse sono le ragioni con le quali si spiega la formazione di bolle d'aria nell'acquifero.

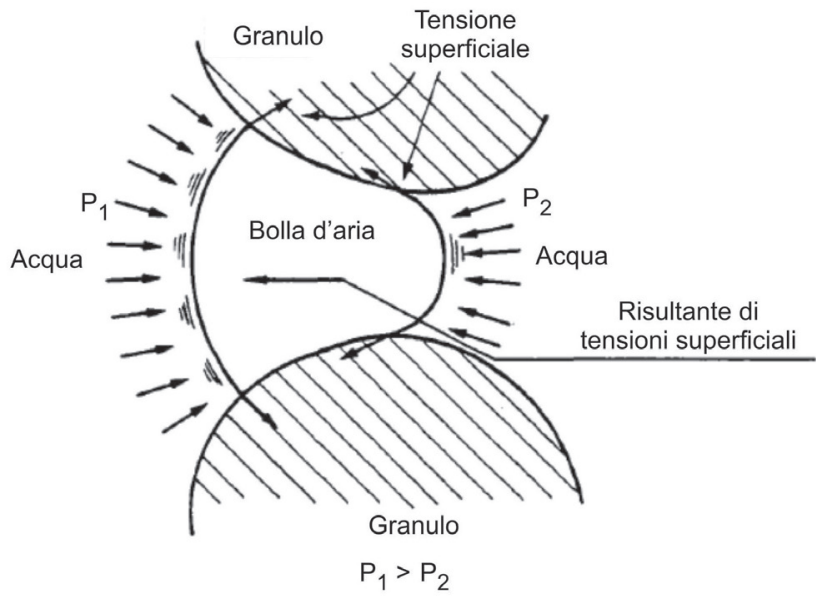

Fig. 4 - Resistance of a gas bubble which, due to deformation, causes the surface tension to produce a resistant force (Olsthoorn 1982).

Fig. 4 - Bolla d'aria presente in un mezzo poroso saturo e resistenza causata da forze di tensione superficiale prodotte dalle deformazioni (Olsthoorn 1982).

\section{Caduta libera di acqua nel pozzo}

Una caduta libera di acqua e una pressione troppo bassa nella parte superiore del tubo di iniezione vengono impediti fornendo quest'ultimo con una corretta limitazione (eventualmente regolabile) alla sua estremità o montando un tubo di iniezione, abbastanza stretto da garantire che il suo attrito di parete fornirà la contropressione richiesta (Sniegocki e Reed 1963).
Con riferimento alla Fig. 5, l'attrito f (resistenza in $\mathrm{m} \mathrm{H} 20$ per $m$ di tubo d'iniezione) è dato dalla relazione:

$$
f=\frac{h}{L}
$$

essendo $\mathrm{h}(\mathrm{m})$ la distanza dal punto più elevato di iniezione dell'acqua rispetto al livello dell'acqua nel pozzo ed L (m) la distanza dal punto più elevato di iniezione e il livello inferiore di iniezione di acqua.

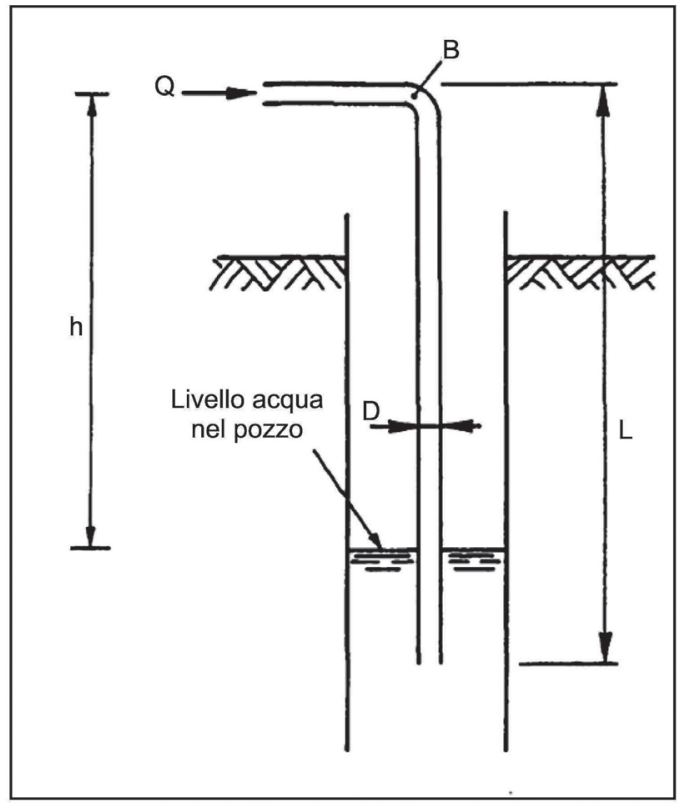

Fig. 5 - Essential measurements of an injection pipe (Olsthoorn 1982).

Fig. 5 - Parametri che influenzano le operazioni di iniezione delle acque nel sottosuolo (Olsthoorn 1982).

\section{Perdite nei condotti nei punti in cui vi è una pressione inferiore a quella atmosferica (valvole, parte superiore della linea di iniezione)}

Appena oltre l'apertura di una valvola strozzata esiste una pressione relativamente bassa. Un valore negativo (inferiore a quello della pressione atmosferica) può comportare l'intrusione di aria. Questo deve essere prevenuto mantenendo un sufficiente livello di pressione a valle della valvola. Il livello della valvola a farfalla $\mathrm{h}_{2}(\mathrm{~m})$ in Fig. 6 rimane positivo quando la testa a valle della valvola $h_{3}(\mathrm{~m})$ soddisfa la seguente relazione

$$
h_{3}>2 \Delta h \sqrt{\frac{v^{2}}{2 g}}
$$

dove $\Delta \mathrm{H}(\mathrm{m})$ è la perdita di carico piezometrico nella valvola, $v(\mathrm{~m} / \mathrm{s})$ la velocità media dell'acqua lungo la valvola e g l'accelerazione di gravità $(\mathrm{N} / \mathrm{kg})$.

Ad una velocità di flusso dell'acqua di $1 \mathrm{~m} / \mathrm{s}$ ed un carico di monte di $\mathrm{h}_{1}$ di $10 \mathrm{~m} \mathrm{H}_{2} \mathrm{O}$, il carico oltre la valvola può quindi 
non scendere sotto $0.91 \mathrm{~m} \mathrm{H}_{2} \mathrm{O}$, come può essere controllato con la precedente relazione.

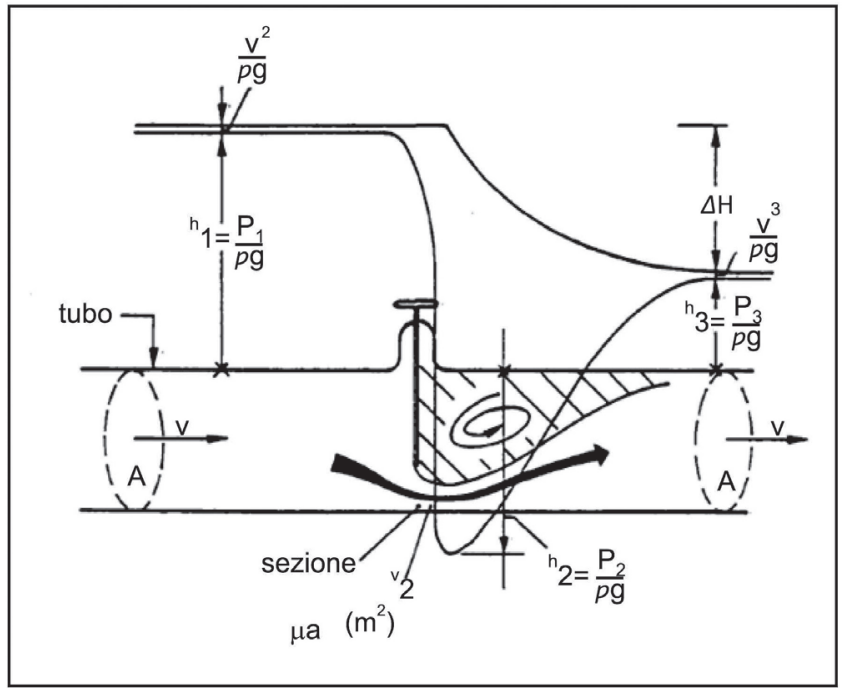

Fig. 6 - Variation of energy and pressure head across a valve (Olsthoorn 1982).

Fig. 6 - Variazione di energia e altezza di pressione lungo una valvola (Olsthoorn 1982)

\section{Sovrasaturazione del gas provocato da caduta di pressione 0 riscaldamento dell'acqua}

Il riscaldamento dell'acqua, che può avvenire in un sistema a ciclo aperto di pompe di calore, diminuisce la solubilità del gas. L'effetto è comunque ridotto in quanto è di circa il $2 \%$ per ogni ${ }^{\circ} \mathrm{C}$.

Al contrario la solubilità aumenta in relazione all'aumento della pressione assoluta e quindi l'effetto provocato dal riscaldamento può essere facilmente compensato da un leggero aumento di pressione.

Un aumento di pressione equivalente a $1 \mathrm{~m} \mathrm{H}_{2} \mathrm{O}$ in un tubo portante con un carico a monte di $5 \mathrm{~m} \mathrm{H}_{2} \mathrm{O}$ (supponendo 10 $\mathrm{m} \mathrm{H}_{2} \mathrm{O}$ di carico come equivalente alla pressione atmosferica) incrementerà la solubilità del 7\%. Questo leggero aumento della pressione è sufficiente dunque per compensare un aumento di temperatura di circa $3.5^{\circ} \mathrm{C}$.
Ciò premesso, la temperatura dell'acqua può non costituire un problema alla formazione di bolle di gas nella ricarica artificiale mediante pozzi di iniezione.

La solubilità del gas è un problema principalmente quando acque sotterranee contenenti naturalmente anche altri gas (come metano, anidride carbonica, etc.) devono essere reimmesse.

A causa del notevole calo di pressione causata dal sollevamento di tali acque, il gas può diventare troppo saturo, con conseguente forte degassaggio con formazione di bolle.

In questi casi è necessario procedere all'installazione di una unità di degassaggio oppure con manutenzione di alta pressione in tutti i tubi e condotti, con costi energetici e di realizzazione di tubazioni resistenti elevati.

\section{Formazione di solidi sospesi}

La formazione di particelle in sospensione rappresenta un problema che assume importanza di seguito a quello della presenza di bolle di gas, almeno a livello temporale.

La presenza di particelle in sospensione e di conseguenza il fenomeno dell'intasamento da sostanze sospese non possono essere sempre completamente evitati in un pozzo.

La concentrazione residua di queste particelle dipende dalla composizione iniziale dell'acqua prelevata e dal trattamento subito, che in generale è assente per quanto attiene alle pompe di calore a circuito aperto; inoltre i fenomeni di corrosione possono mettere in circolo altre sostanze nel pozzo.

Le dimensioni delle particelle presenti nell'acqua sono di diversi ordini di grandezza differenti come illustrato nella Fig. 7, insieme all'indicazione delle dimensioni di particelle di sostanze solide e di microrganismi sono indicate anche $\mathrm{i}$ processi fisici applicabili per la loro rimozione.

Il fenomeno dell'intasamento da solidi sospesi viene interpretato per similitudine con quello relativo alle membrane che avviene in tre fasi come illustrato nella Fig. 7.

$\mathrm{Si}$ incomincia con un addensamento dei materiali solidi in vicinanza del filtro del pozzo, per proseguire formando una specie di panello di fango o di gel che poi può essere forzato ad entrare in tutto il dreno e nell'acquifero dal moto dell'acqua di ricarica.

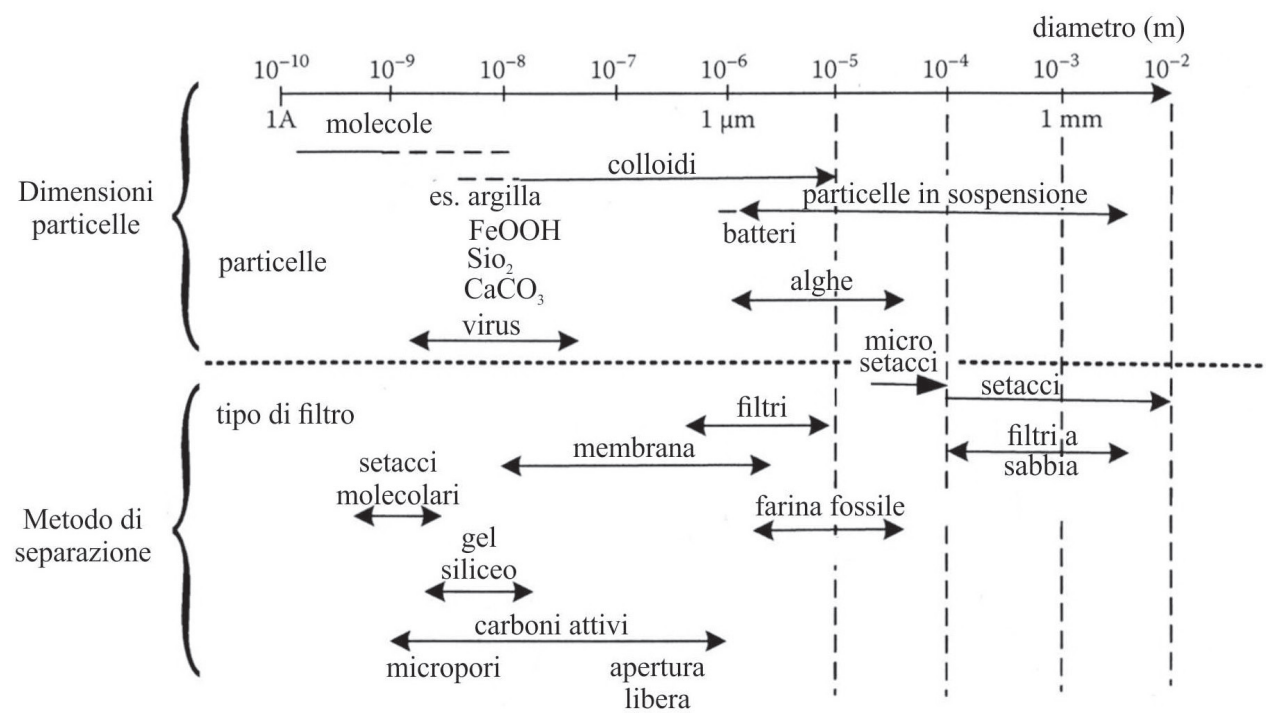

Fig. 7 - Particle sizes to be seen in natural water and groundwater and physical processes for separation of particles (adapted with permission from Stumm, Chemical interaction separation, American Chemical Society, Nov 1,1977, Copyright (C)1977, American Chemical Society).

Fig. 7 - Dimensioni delle particelle presenti nelle acque superficiali e sotterranee e processi fisici per la loro rimozione (adattata con il permesso di Stumm 1977, Chemical interaction separation, American Chemical Society, Nov 1,1977, Copyright (C1977, American Chemical Society). 


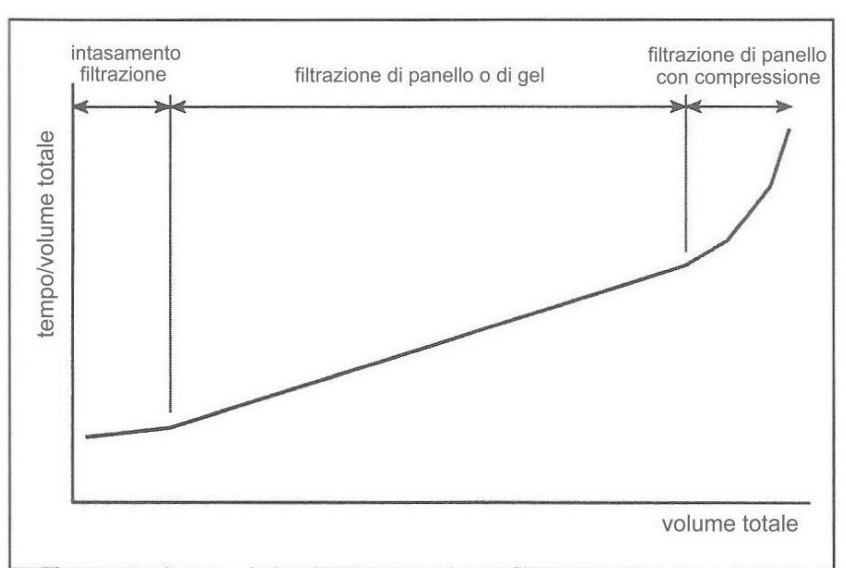

Fig. 8 - Stages of clogging on a membrane filter (similarity with the well clogging) from Pyne 2005.

Fig. 8 - Fasi di intasamento di una membrana (similitudine con l'intasamento di un pozzo) da Pyne 2005

In contrasto con la caratteristica tipica delle acque sotterranee, che hanno una temperatura $\mathrm{T}_{\mathrm{w}}$ e una viscosità $\mu_{\mathrm{w}}$, costanti, questi parametri si modificano e devono essere presi in considerazione per le pompe di calore a circuito aperto.

La differenza di temperature tra acque iniettate in inverno $\left(2{ }^{\circ} \mathrm{C}\right)$ e in estate $\left(28^{\circ} \mathrm{C}\right)$ in un impianto di pompe di calore a circuito aperto, che funziona come riscaldamento invernale e come condizionamento estivo di edifici, determina una diversa resistenza che si incrementa di circa il $100 \%$.

La viscosità dell'acqua $\mu_{\mathrm{w}}\left(\mathrm{Ns} / \mathrm{m}^{2}=\mathrm{Pa}-\mathrm{s}\right)$ segue una relazione con la temperatura che può essere approssimata dall'espressione:

$$
\mu_{w}=\left[\left(\frac{510}{T_{w}+43.1}\right)^{1.502}\right] \times 10^{-3}
$$

valida per $0<\mathrm{T}_{\mathrm{w}}<100^{\circ} \mathrm{C}$.

L'effettivo intasamento di un pozzo di iniezione deve essere considerato adottando una correzione della temperatura, con riferimento ad un valore standard di $10{ }^{\circ} \mathrm{C}$ come segue:

$$
M=\frac{\mu_{10}}{\mu_{T}}=\frac{T+20}{30}
$$

La resistenza all'iniezione in un pozzo a $10{ }^{\circ} \mathrm{C}$ denominata W10 $\left(\mathrm{m} /\left(\mathrm{m}^{3} / \mathrm{h}\right)\right.$ o in sintesi $\left.\mathrm{h} / \mathrm{m}^{2}\right)$ viene calcolata dopo la correzione per la temperature per l'aumento di pressione o risalita di livello piezometrico $\mathrm{h}(\mathrm{m})$ per unità di portata di iniezione $\mathrm{Q}\left(\mathrm{m}^{3} / \mathrm{h}\right)$.

La resistenza nel pozzo dovuta alla presenza di intasamento $\mathrm{W}_{\mathrm{C}_{10}}$ è costituita dalla differenza tra la resistenza complessiva $\mathrm{e}$ la resistenza naturale del pozzo (quando è nuovo) ed è calcolata come:

$$
W_{c_{10}}=M \frac{h_{w}-h_{r}}{Q}
$$

essendo hw ed $\mathrm{hr}(\mathrm{m})$ rispettivamente il livello nel pozzo e il livello in un piezometro immediatamente adiacente o nell'acquifero indisturbato.
Uno schema del comportamento delle acque iniettate in un pozzo di iniezione interessato da intasamento è illustrato in Fig. 9.

Se è stato realizzato un tubetto piezometrico nel dreno come attualmente avviene, possono essere determinati separatamente l'intasamento nel filtri e quello nei primi centimetri del dreno.

Poiché nella maggioranza dei casi l'intasamento tende a concentrarsi sulla parete del perforo e penetra al massimo per qualche centimetro nel circostante acquifero, anche il livello in un tubetto piezometrico nello stesso perforo, ma nel dreno, può essere utilizzato come riferimento per la formazione non intasata.

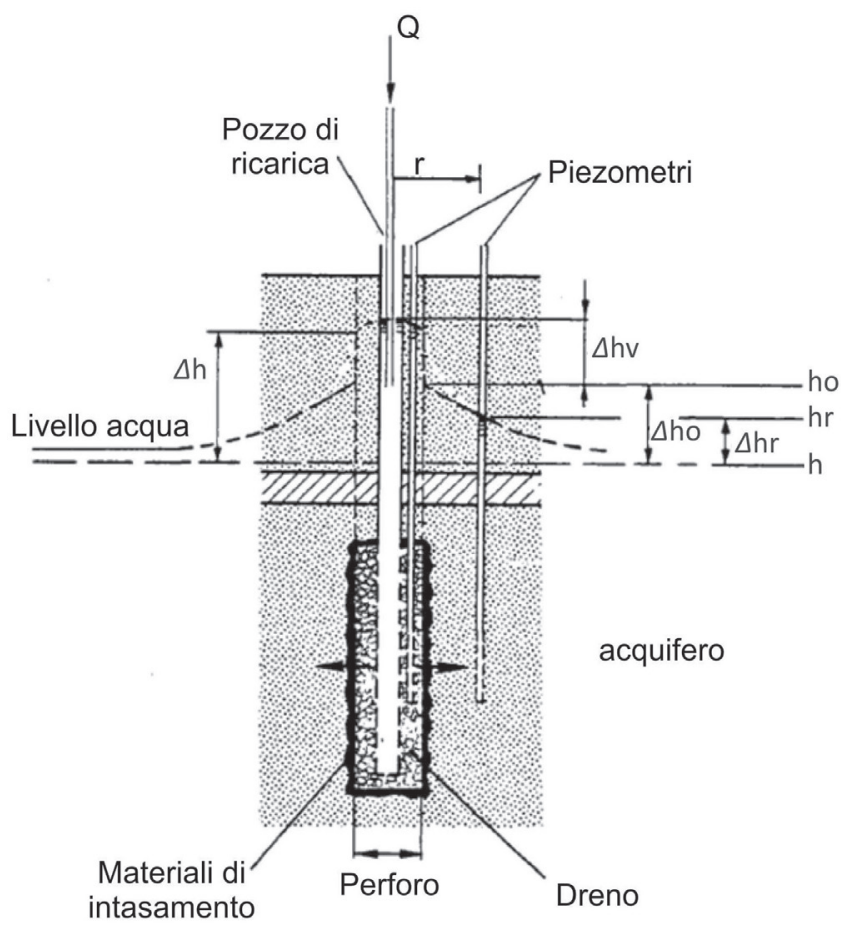

Fig. 9 - Clogging of an recharge well and relevant water levels heads in the surrounding piezometer (Olsthoorn 1982).

Fig. 9 - Schema delle variazioni di livello piezometrico in un pozzo di iniezione con intasamento e nei piezometri circostanti (Olsthoorn 1982).

I costituenti del materiali in sospensione nell'acqua possono essere di diversa natura, forma e dimensione, organici o inorganici nella composizione.

Se la composizione di tale materiale è abbastanza costante, di solito si genera un notevole aumento della resistenza per $\mathrm{kg}$ di materiale depositato e se inoltre la concentrazione è costante allora il rapporto tra resistenza all'intasamento e il volume totale dell'acqua iniettata è lineare.

Oltre il normale rapporto tra l'aumento della pressione e la portata secondo la legge di Darcy, anche la fornitura di materiale di intasamento per unità di tempo è proporzionale alla portata.

Esiste una relazione quadratica ricostruita mediante dati sperimentali fra il tasso al quale la pressione di iniezione o il livello dell'acqua $\mathrm{h}(\mathrm{m})$ sale nel pozzo e la portata $\mathrm{d}$ iniezione 
$\mathrm{Q}\left(\mathrm{m}^{3} / \mathrm{s}\right)$ tale che:

$$
\frac{\delta h}{\delta t} \sim Q^{2}
$$

In questa relazione l'accumulo di pressione misurato può essere convertito in una certa portata standard ed essere tracciato rispetto al volume totale iniettato di acqua e la relazione ottenuta da sperimentazioni è lineare (Sniegocki 1963).

Le acque e i litotipi costituenti l'acquifero sono molto differenti e ad esempio le proprietà di intasamento delle sospensioni di argilla si differenziano per le argille provenienti da diverse fonti, così come per una sospensione di idrossidi di Ferro si ha una importante dipendenza dal valore del $\mathrm{pH}$ dell'acqua.

Talvolta il tasso di intasamento non si attenua, nonostante una riduzione marcata della concentrazione di sostanze sospese. Questo è stato spiegato considerando la reazione di coagulazione delle particelle, in quanto si rimuove la carica negativa sulle particelle in sospensione che vengono così trattenute molto più efficientemente dai granuli dell'acquifero carichi negativamente, non esistendo repulsione tra le stesse cariche. In questo modo, le particelle diventano concentrate in un sottile strato di intasamento, con un conseguente aumento della resistenza al flusso.

Se la filtrazione dal suolo è altamente efficiente, pochi grammi di materia in sospensione per $\mathrm{m}^{2}$ di area di ricarica sono sufficienti a causare un aumento del livello dell'acqua nel pozzo, per un'aliquota di ricarica di circa $1 \mathrm{~m} / \mathrm{h}$ rispetto alla superficie della parete del foro.

Ad una elevata concentrazione di $1 \mathrm{mg} / \mathrm{l}$, questa situazione si può verificare in poche ore. Dove la filtrazione dal suolo è meno efficiente, in presenza di materiale fine caricato negativamente delle particelle in sospensione o materiale grossolano, il processo può richiedere molto più tempo (Rahman et al. 1969).

Il coinvolgimento del dreno che circonda il filtro ben corrisponde a quella del primo strato grossolano di un filtro multi-letto.

Il dreno intrappola le particelle senza apprezzabile aumento della resistenza, alleviando in tal modo la formazione sulla parete dove si concentra l'intasamento.

Generalmente, il dreno fornisce una riduzione sostanziale dell'aliquota di intasamento.

$\mathrm{Da}$ quanto sopra illustrato sembra che, basandosi esclusivamente su analisi delle acque di iniezione e delle particelle in sospensione in esse contenute, si possa prevedere con successo l'intasamento dei pozzi di iniezione in fase progettuale.

Ma l'indagine è ulteriormente ostacolata dal fatto che un'acqua con una bassa concentrazione di solidi sospesi come ad esempio un'acqua potabile, non esiste alcuna relazione tra la concentrazione, la torbidità e l'intasamento di un pozzo di iniezione. Di conseguenza è difficilmente possibile trarre conclusioni pertinenti relativi alla previsione degli effetti indotti dall'iniezione di acque e del relativo fenomeno di intasamento.
Migliori risultati sono ottenuti da una prova di filtro a membrana ideata da Schippers e Verdouw 1980 forzando l'acqua in esame sotto pressione costante ( 2 bar) attraverso un filtro a membrana di $47 \mathrm{~mm}$ di diametro con micropori di $0,45 \mu \mathrm{m}$.

Il risultato è ridotto a quello che viene chiamato un indice modificato di filtro MFI («Modified Fouling Index») espresso in $\mathrm{s} / \mathrm{l}^{2}$.

Il parametro MFI rappresenta la pendenza della rette che interpola i dati sperimentali di il tempo/volume di acqua in scala aritmetica e, a causa di tempi di prova e volumi ridotti, l'unità di misura è $s / 1^{2}$.

I valori di MPI determinati devono essere normalizzati rispetto a condizioni standard di una pressione $\mathrm{P}=200 \mathrm{kPa}$ che sono espresse mediante la relazione:

$$
M F I_{\text {norm }}=M F I \cdot \frac{\mu_{20}}{\mu} \cdot \frac{P}{200}
$$

essendo $\mu_{20}$ e $\mu$ la viscosità dell'acqua rispettivamente alla temperatura standard e secondo la misura sperimentale.

Per pozzi di iniezione MFI con valori inferiori a 3 sono positivi e superiori a 10-15 negativi.

Per arrivare ad un progetto definitivo rimane necessaria una sperimentazione, interpretando i dati provenienti da esperimenti con i pozzi di prova.

Laumento di $\Delta \mathrm{h}$ in testa ad un pozzo di iniezione al di sopra del livello statico (o al di sopra del livello nel piezometro limitrofo) è divisibile in una porzione $\Delta \mathrm{h}_{0}$ risultante dall'acquifero ed una porzione $\Delta \mathrm{hv}$ causato da intasamento, che ha ridotto la permeabilità $\mathrm{k}$ tra le distanze $\mathrm{r}_{0}$ e $\mathrm{r}_{\mathrm{v}}$ a $\mathrm{k}_{\mathrm{v}}$. Nel caso del pozzo di iniezione, l'incremento totale di altezza sarà il seguente:

$$
\begin{aligned}
& \Delta h_{0}=\frac{Q}{2 \pi K h} \ln \left(\frac{R}{r_{0}}\right) \\
& \Delta h_{v}=\frac{Q}{2 \pi K^{*} h} \ln \left(\frac{r_{v}}{r_{0}}\right)=\frac{Q}{2 \pi h}\left(\frac{1}{K_{v}}-\frac{1}{K}\right) \ln \left(\frac{r_{v}}{r_{0}}\right) \\
& K^{*}=\frac{K K_{v}}{K-K_{v}} \sim K_{v}
\end{aligned}
$$

essendo generalmente $\mathrm{K}>\mathrm{K}_{\mathrm{v}}$.

Come è noto la conducibilità idraulica $\mathrm{k}(\mathrm{m} / \mathrm{s})$ e la permeabilità intrinseca $\mathrm{k}\left(\mathrm{m}^{2}\right)$ sono in relazione come segue:

$$
K=\frac{\rho g}{\mu} k
$$

La caduta di pressione $\mathrm{dp}\left(\mathrm{N} / \mathrm{m}^{2}\right)$ risultante dal flusso attraverso il livello intasato di spessore $\mathrm{dl}(\mathrm{m})$ e permeabilità $\mathrm{k}\left(\mathrm{m}^{2}\right)$, con flusso $\mathrm{Q}\left(\mathrm{m}^{3} / \mathrm{s}\right)$ e superficie filtrante A $\left(\mathrm{m}^{2}\right)$, segue 
la relazione:

$$
d p=\frac{\mu Q}{k A} d l
$$

Si introduce poi il concetto di resistenza $\mathrm{W}$ come espressa da:

$$
d W=d\left(\frac{p}{Q \mu}\right)=\left(\frac{l}{k A}\right) d l
$$

Il volume del livello intasante $\mathrm{dV}$ portato all'interno considerato per $\mathrm{m}^{3}$ di acqua è in relazione ad A come:

$$
d V=A d l
$$

e quindi nel pozzo:

$$
d V=(2 \pi r H) d r
$$

In cui $r(m)$ è la distanza dal centro del pozzo dal punto in cui si ha l'intasamento e $\mathrm{H}(\mathrm{m})$ la lunghezza del dreno in modo che:

$$
d W=\left(\frac{d V}{k A^{2}}\right)=\left(\frac{l}{2 \pi k H}\right) \frac{d r}{r}
$$

Nel caso di presenza di panello nel perforo come in Fig. 10, $r$ decresce ulteriormente in modo costante $\left(r<r_{0}\right)$ :

$$
V=\int_{0}^{t} Q C d t=\pi H\left(r_{0}^{2}-r^{2}\right)
$$

in cui $\mathrm{C}$ è la concentrazione dei solidi sospesi espresso in termini di $\mathrm{m}^{3}$ di livello intasante per $\mathrm{m}^{3}$ di acqua.

Nel caso di filtrazione profonda in Fig. 10, la (17) viene applicata in modo inverso $\left(r>r_{0}\right)$ :

$$
V=\int_{0}^{t} Q C d t=\pi H\left(r^{2}-r_{0}^{2}\right)
$$

Per:

$$
h=\frac{p}{\rho g}
$$

si ha:

$$
\Delta h_{v}=\left(\frac{Q}{2 \pi k H}\right)\left(\frac{\mu}{\rho g}\right) \ln \left(1 \pm \frac{v}{\pi r_{0}^{2} H}\right)
$$

dove il segno positivo si applica alla filtrazione profonda e il segno negativo alla filtrazione nel panello (altro processo atteso con altra permeabilità).

Per un breve intervallo di tempo e quindi con un piccolo V, il rapporto va oltre a quello per filtrazione lineari:

$$
\Delta h_{v}=\left(\frac{l}{\rho g} \frac{\mu}{k}\right)\left(\frac{Q V}{A_{0}^{2}}\right)=\left(\frac{l}{\rho g} \frac{\mu}{k}\right) v V^{*}
$$

$$
A_{0}=2 \pi r_{0} H
$$

essendo v l'aliquota di infiltrazione nel perforo $(\mathrm{m} / \mathrm{h})$ e $\mathrm{V}^{*}$ $(\mathrm{m})$ il volume di solidi sospesi introdotto per $\mathrm{m}^{2}$ di area del perforo.

Poiché questo rapporto è derivato indipendentemente dalla portata, si applica in generale per questo tipo di intasamento e può essere utilizzato indifferentemente per infiltrazione sotto pressione costante o sotto costante portata.

Con una concentrazione costante $\mathrm{C}$ si ha la seguente relazione:

$$
\Delta h_{v}=\left(\frac{l}{\rho g} \frac{c \mu}{k}\right) v U^{*}
$$

dove $\mathrm{U}^{*}$ rappresenta il volume totale di acqua infiltrata $(\mathrm{m})$ per $\mathrm{m}^{2}$ di area del perforo.

Se anche tale portata può essere costante si ha:

$$
\Delta h_{v}=\left(\frac{l}{\rho g} \frac{c \mu}{k}\right) v^{2} t
$$

che è equivalente alla precedentemente considerata relazione quadratica tra incremento di pressione e aliquota di infiltrazione.

A pressione $\mathrm{p}$ o carico $\mathrm{h}$ costanti e con un volume totale infiltrato $\mathrm{U}^{*}$ per $\mathrm{m}^{2}$ di area del perforo e con $\mathrm{v}=\mathrm{v}_{0}$ se $\mathrm{t}=0$ si ha:

$$
\begin{aligned}
& v=\frac{v_{0}}{a \cdot 1} \\
& a=\left(\frac{v_{0}}{p} \frac{c \mu}{k}\right) U^{*}
\end{aligned}
$$

Esplicitando rispetto al tempo si ha invece:

$$
\begin{aligned}
& v=\frac{v_{0}}{\sqrt{b t * 1}} \\
& b=\left(\frac{2 c \mu}{p k}\right)
\end{aligned}
$$

Le formule sopra riportate possono essere utilizzati per descrivere l'intasamento da solidi in sospensione. La qualità dell'acqua effettiva, che può essere identificata con il fattore $\mathrm{C} / \mathrm{k}$, può essere determinato solo sperimentalmente e varierà in maniera sconosciuta in un'acqua naturale, mentre $\mathrm{C} \mathrm{e} \mathrm{k}$ dipendono anche dai litotipi della formazione.

Una considerazione importante è che le formule consentono di confrontare l'intasamento sperimentato in differenti pozzi. A tal fine l'aumento della pressione in testa pozzo viene ridotto al numero di metri di innalzamento del carico dell'acqua che sarebbe verificato se l'acqua fosse stata infiltrata ad una temperatura di $10{ }^{\circ} \mathrm{C}$ per 1 anno ad un tasso di $1 \mathrm{~m} / \mathrm{h}$ rispetto all'area del perforo. 
A tale proposito è sufficiente solo confrontare l'inizio e la fine del periodo considerato. All'inizio del periodo considerato la portata è $\mathrm{Q}_{\mathrm{b}}, \mu_{\mathrm{b}}$ la viscosità dell'acqua e il carico idraulico in regime statico del pozzo o in un vicino tubetto piezometrico $h_{b}$. Alla fine del periodo considerato questi quantitativi saranno $Q_{e}, \mu_{e}$ e $h_{e}$.. Durante il periodo di durata $t$, un volume di acqua $U$ viene iniettato. Il pozzo ha una superficie di infiltrazione A. Laltezza richiesta di iniezione standard $\Delta \mathrm{h}_{\mathrm{s}}$ che potrebbe sopravvenire al tasso di infiltrazione standard $\mathrm{v}_{0}=\mathrm{Q}_{0} / \mathrm{A}=1 \mathrm{~m} / \mathrm{h}$, la viscosità dell'acqua $\mu_{10}$ (temperatura dell'acqua $10{ }^{\circ} \mathrm{C}$ per il periodo di tempo standard t di un anno (8760 h) ammonta a:

$$
\Delta h_{s}=\left(h_{e} \frac{\mu_{0} v_{0}}{\mu_{e} Q_{e} / A}-h_{b} \frac{\mu_{0} v_{0}}{\mu_{e} Q_{e} / A}\right)\left(\frac{v_{0} t_{0}}{U / A}\right)
$$

se $\mu_{\mathrm{e}}=\mu_{\mathrm{b}}=\mu_{0}, \mathrm{Q}_{\mathrm{e}}=\mathrm{Q}_{\mathrm{b}}=\mathrm{v} . \mathrm{A}$ e $\mathrm{U}=\mathrm{v} A \mathrm{t}$, si ha la nota relazione quadratica:

$$
\Delta h_{s}=\left(h_{e}-h_{b}\right)\left(\frac{v_{0}}{v}\right)^{2}\left(\frac{t_{0}}{t}\right)
$$

In questo modo dati di pozzi di iniezione di origine diversa e in diversi luoghi del mondo, posti all'interno di acquiferi differenti, possono essere confrontati ricavandone indicazioni circa la loro efficienza.

L'importanza della presenza di solidi sospesi sul flusso delle acque in arrivo ad un pozzo è riassunta nella Fig. 11, dalla quale è possibile osservare come durante la sperimentazione si sia avuta una riduzione significativa, molto spesso oltre il $50 \%$ per valori di $0.2 \mathrm{mg} / \mathrm{L}$ solidi sospesi per $\mathrm{cm}^{2}$ di superficie di sabbia.

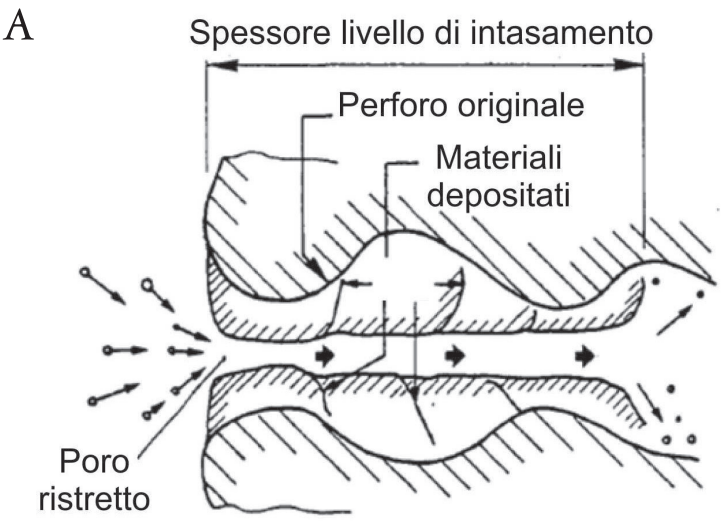

B

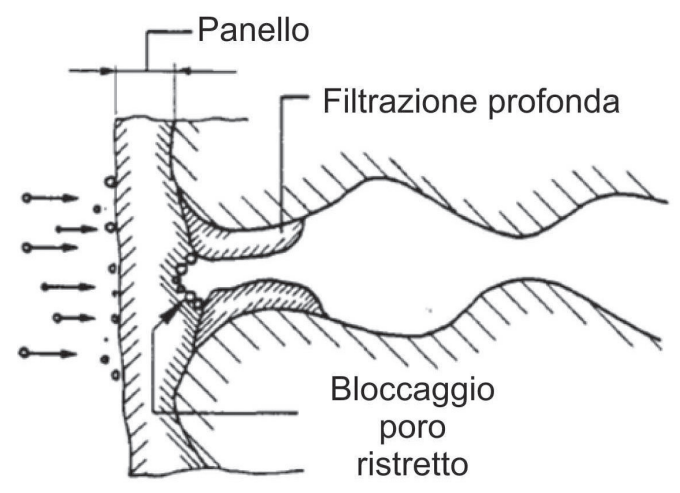

Fig. 10 - Scheme of well clogging: A) clogging in deep-bed filtration, B) clogging will occur through cake filtration (Olsthoorn 1982).

Fig. 10 - Schemi di intasamento di un pozzo: A-senza panello e infiltrazione profonda, B-con panello non completamente eliminato e filtrazione superficiale continua (Olsthoorn 1982)

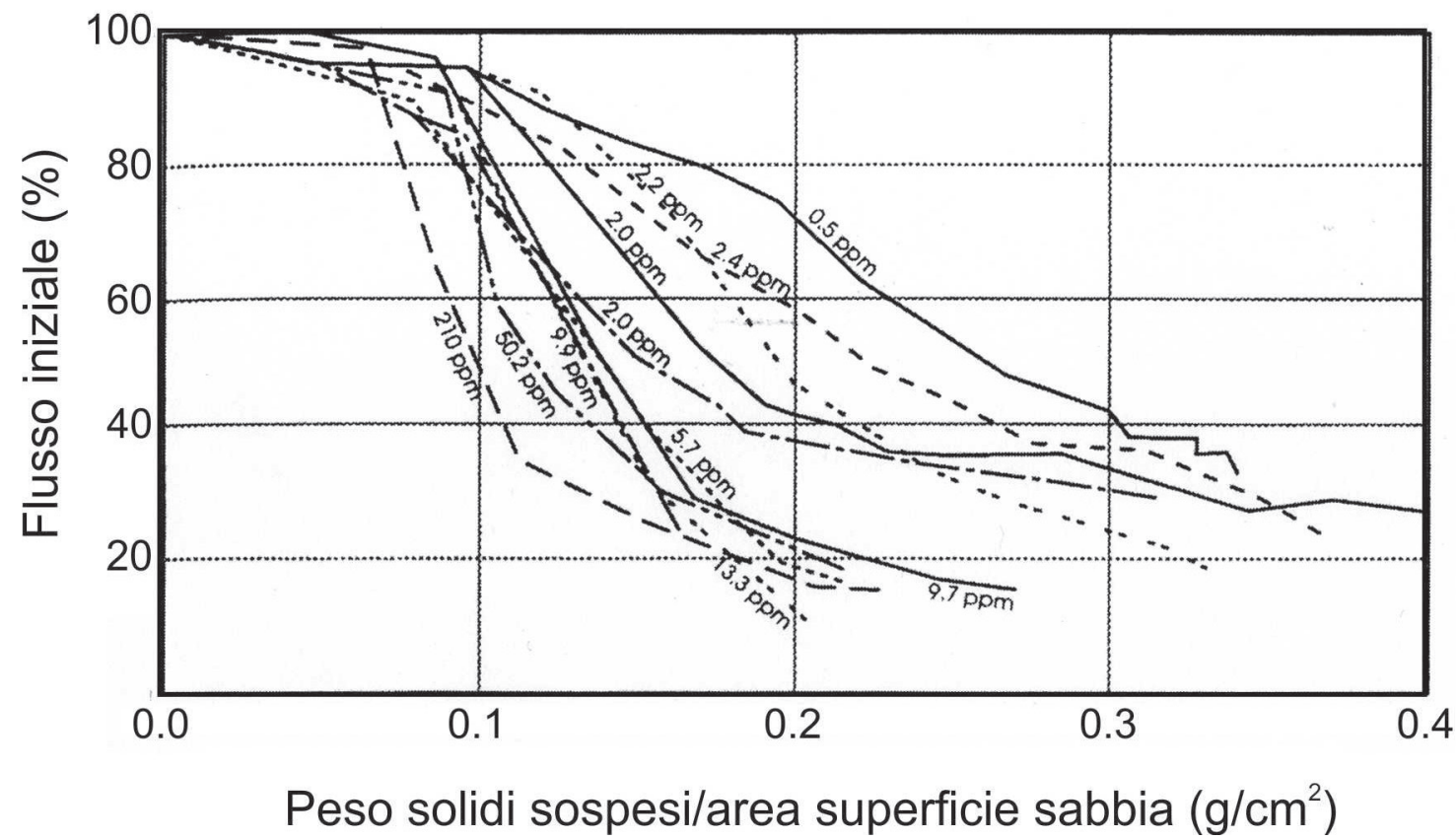

Fig. 11 - Effect of different concentrations of suspended solids on the recharge operations (Bichara 1986, with permission of The American Society of Civil Engineers - ASCE).

Fig. 11 - Effetto di differenti concentrazioni di solidi sospesi sulle operazioni di ricarica (Bichara 1986, permesso di riproduzione The American Society of Civil Engineers - ASCE). 
Si sottolinea infine l'esigenza di disporre di acqua di ricarica senza solidi sospesi e di minimizzarne l'eventuale produzione nel corso della gestione del pozzo di resa.

Infatti durante il pompaggio in un pozzo di prelievo sono continuamente rimosse le particelle solide provenienti dall'acquifero, mentre in un pozzo di ricarica le eventuali particelle contenute nelle acque di ricarica sono continuamente aggiunte ai filtri e al dreno, determinando un progressivo peggioramento della situazione.

\section{Crescita batterica}

La crescita batterica determina la formazione di "biofouling", che è costituito da un film di microrganismi in corrispondenza dei filtri che ostacola il flusso idrico e la sua formazione avviene in diverse fasi di seguito riassunte.

L'entità e la velocità di tale crescita dipende anche dalla presenza di nutrienti nelle acque di ricarica.

Fase 1- I batteri hanno dimensioni tipicamente molto piccole, dell'ordine di $1-2 \mu \mathrm{m}$, anche se sono stati riconosciuti batteri di dimensioni maggiori.

La maggior parte dei batteri preferisce attaccarsi ad una superficie e l'acquifero offre una superficie dei granuli molto elevata con carica positiva per l'attacco dei microrgamismi.

A titolo di chiarificazione si osserva che la superficie $A\left(\mathrm{~m}^{2}\right)$ di un granulo sferico di volume $\mathrm{V}\left(\mathrm{m}^{3}\right)$ e raggio $\mathrm{r}(\mathrm{m})$ è data da:

$$
A=\frac{3}{r} V
$$

Pertanto più piccola è la dimensione del microrgamismo, maggiore è il rapporto tra area e volume da esso occupati.

I microrganismi aderiscono al supporto poroso attraverso interazioni elettrostatiche, forze di idratazione e interazioni idrofobiche e steriche. Questa adesione può essere influenzata da fattori quali carica di minerali e batteri di superficie, rugosità della superficie dei minerale, formazione esopolimeri, predazione e concorrenza. I batteri aderiscono anche ai filtri e al dreno, che hanno rapporti inferiori superficie/volume rispetto ai litotipi dell'acquifero e quindi tendono a sporcarsi rapidamente, determinando l'intasamento del pozzo.

Fase 2- Una volta adesi i batteri si riproducono e crescono formando colonie. Le colonie crescono verso l'esterno per formare un biofilm secernendo esopolisaccaridi (EPS), una matrice che origina ritenzione idrica. La composizione e la struttura dei EPS, così come le loro proprietà fisiche e chimiche, possono variare ampiamente e dipendono dalla composizione della comunità microbica e dalla presenza di ioni in soluzione. Diversi ceppi microbici crescono fino a formare una comunità o un biofilm consortile (Alford G., Cullimore R. 1999). Un biofilm può essere visto come un gel polimero organico formato da microrganismi che vivono intrappolati all'interno (Fig. 12). La natura del gel può influenzare il calore e le proprietà del trasporto di massa, condizionando così l'efficacia dei biocidi eventualmente utilizzati per la pulizia dei filtri (Characklis e Marshall 1990).

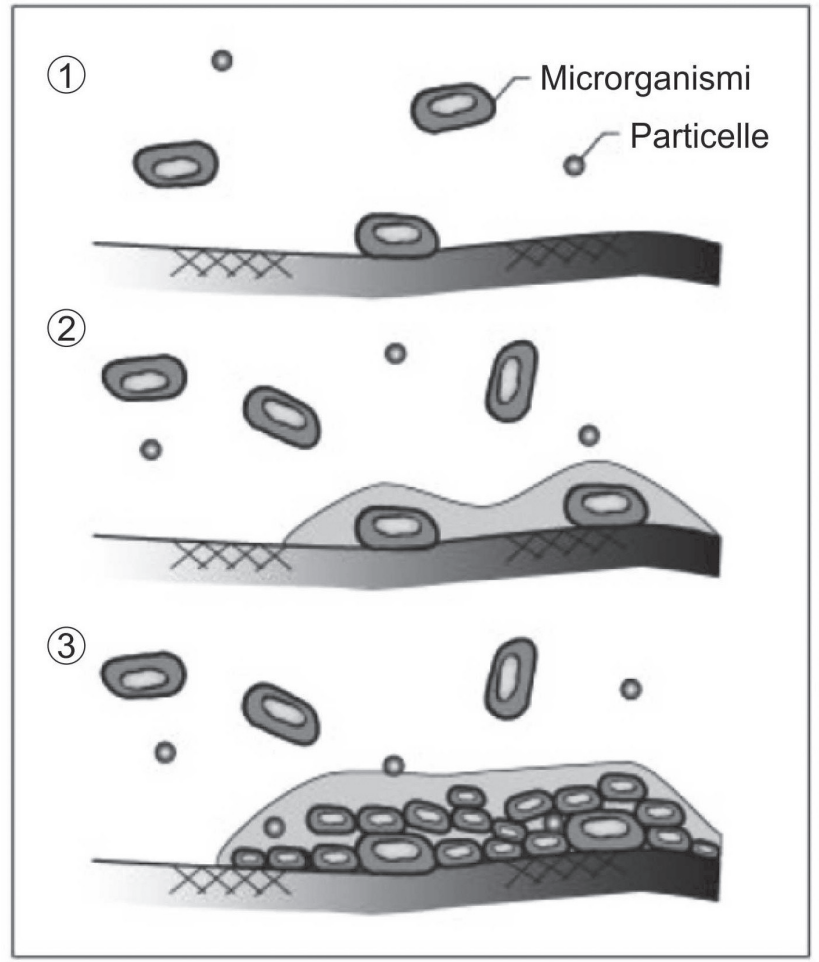

Fig. 12 - Stages of evolution of bacteria growth and biofilm formation: 1 - bacteria attach to particle surface via physical and chemical interaction reaction, 2 - attached bacteria synthesize and excrete exapolysaccarides (EPS matrix of biofilm), 3 - attached bacteria growth and divide: biofilm matrix includes water, exapolysaccarides, lipids, proteins, solids (clays, colloids), cations (especially $\mathrm{Ca2}^{+}$) ((Strategic Environmental Security Technology Certification Program ESTCP, 2005).

Fig. 12 - Fasi di evoluzione della crescita batterica e formazione di un biofilm: 1- batteri che si attaccano a superficie per reazioni chimicofisiche, 2-batteri adesi che si riproducono e secernono esapolisaccaridi (EPS matrice del biofilm), 3- batteri adesi che crescono e si dividono in: matrice del biofilm inclusa l'acqua, esapolissaccaridi, lipidi, proteine, solidi (argille, colloidi) cationi (in modo particolare $\mathrm{Ca}^{2+}$ ) ((Strategic Environmental Security Technology Certification Program - ESTCP, 2005).

Fase 3- La presenza di cationi polivalenti (ad esempio, $\mathrm{Mg}^{2+}$, e $\mathrm{Ca}^{+2}$ ) migliora la resistenza meccanica del biofilm, consentendo di sopportare notevoli sforzi di taglio (Mayer et al. 1999). Il biofilm può indurire attraverso il bioaccumulo di Ferro e altri cationi metallici quali $\mathrm{Mn}, \mathrm{Al}$, Cu e Zn (Cullimore 1999; Smith 1995) e attraverso la sintesi di strutture cristalline di solito a base di carbonati. Durante questo processo vi è anche l'intrappolamento di argille, limi e sabbie. Nei sistemi biofilm anaerobici, batteri solforiduttore possono generare idrogeno solforato, che può reagire con il Fe e Mn per formare solfuri metallici che possono infiltrarsi nella matrice del biofilm. Batteri metanigeni possono produrre metano e anche anidride carbonica, che possono essere intrappolati nel biofilm, oppure i gas possono migrare ed occludere i pori nella matrice dell'acquifero, secondo i meccanismi precedentemente illustrati relativi alle bolle di gas.

Fase 4 - A causa degli sforzi di taglio provocati dal movimento dell'acqua, la biomassa adesa può essere staccata dal biofilm e trasportata dalla fase acquosa in un'altra posizione, dove si può ricollegare e ciò può ridistribuire 
sostanzialmente biomassa in mezzi porosi distanti dalla posizione di formazione (Taylor e Jaffe 1991).

Fase 5 - Alla fine il biofilm ha una massa sufficientemente grande che occupa una parte significativa del volume vuoto del filtro, del dreno o della matrice dell'acquifero e di conseguenza di verifica l'ostruzione o l'occlusione dei pori, con conseguente diminuzione delle prestazioni del pozzo.

Non è possibile considerare la grande varietà di composizione e anche la diversa concentrazione di elementi e composti che influenzano la presenza di comunità di microrganismi nelle acque sotterranee.

Uno dei maggiori problemi è costituito dalla presenza di Ferrobatteri che sono così definiti come il gruppo di batteri aerobici che utilizzano l'ossidazione di ioni di Ferro e/o di Manganese come componente essenziale nel loro metabolismo.

Spesso una consistente crescita di questi batteri costituisce un substrato per altri batteri che possono degradare i materiali in condizioni anaerobiche, per formare prodotti quali idrogeno solforato e altri acidi.

Alcuni generi di batteri provocano l'Ossidazione di Ferro e/o Manganese e per il Ferro si hanno ad esempio Lieskella, Gallionella e Tiobacillus feroxidans (Cullimore 1978).

La loro crescita è generalmente favorita dai seguenti fattori:

- presenza di Ferro in soluzione con valori maggiori di 0.2-0.5 mg/l e di Manganese superiori a $0.05 \mathrm{mg} / \mathrm{l}$ con concentrazioni che continuano a rinnovarsi nel pozzo in seguito al flusso delle acque sotterranee;

- ad eccezione di Thiobacillus ferrooxidans, la crescita batterica avviene nell'intervallo di $\mathrm{pH}$ di of 5.4 a 7.2;

- la crescita avviene con un contenuto di Ossigeno disciolto nelle acque inferiore a $5 \mathrm{mg} / \mathrm{l}$;

- l'effetto della temperatura non è definito nel dettaglio e si è rilevato ad esempio che per Sphaerotilus si ha un valore minimo di $15^{\circ} \mathrm{C}$ e ottimale di $25-30^{\circ} \mathrm{C}$, per Leptothrix minimo di $10^{\circ} \mathrm{C}$ e ottimale di $20-25^{\circ} \mathrm{C}$, per Crenothrix minimo di $6^{\circ} \mathrm{C}$ e ottimale di $26-28^{\circ} \mathrm{C}$ e per Thiobacillus ferrooxidans ottimale di $15-20^{\circ} \mathrm{C}$.

Un indicatore di possibilità di intasamento dovuto a batteri è il contenuto di Carbonio Organico Assimilabile (AOC) nelle acque di infiltrazione in quanto è in relazione al carico organico di un'acqua e descrive il potenziale di crescita per i microrganismi. La concentrazione di AOC deve essere $<10$ $\mu \mathrm{g}$ acetato-C/l, in modo da ridurre fortemente la probabilità di intasamento in un pozzo di ricarica dovuto alla crescita batterica (Van der Kooij et al. 1982).

Una sintesi circa i fattori che influenzano l'intasamento dovuto alla crescita batterica è inoltre riportato in Fig. 13, in quanto nell'acqua sono presenti diverse sostanze che in combinazione tra loro determinano l'innesco e il procedere dei fenomeni di crescita batterica responsabili dell'intasamento.

In presenza di consistenti problemi dovuti alla crescita batterica nel pozzo, si è adottato in un caso un trattamento di disinfezione delle acque mediante UV prima della reimmissione, di costo significativo; vista la tipologia, questo trattamento potrebbe avere maggiore accettabilità in termini autorizzativi.

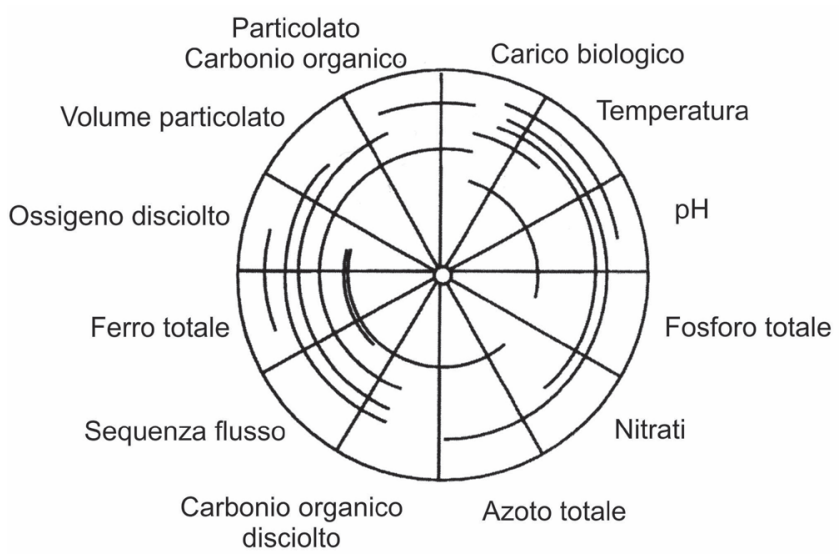

Fig. 13 - Factors affecting bacterial growth and associated clogging of a well (Pyne 2005).

Fig. 13 - Fattori che influenzano la crescita batterica e che determinano l'intasamento di un pozzo (Pyne 2005).

\section{Reazioni chimico-fisiche}

Quando le acque sotterranee prelevate da un pozzo sono portate in superficie possono verificarsi una serie di processi chimici e reazioni tra cui:

1. degassaggio $\mathrm{CO}_{2}$ e associato aumento del $\mathrm{pH}$ e precipitazione di minerali (ad esempio calcite);

2. dissoluzione $\mathrm{O}_{2}$ nelle acque sotterranee e ossidazione dei metalli disciolti formando precipitati poco solubili;

3. formazione di biofilm conseguente ad arricchimento di $\mathrm{O}_{2}$ delle acque.

Pur essendo l'acqua restituita nello stesso acquifero da cui viene prelevata, il contenuto di sostanze solide e in sospensione possono differire tra l'estratto e il reiniettato; ad esempio nelle acque potabili prelevate da pozzi giudicati efficienti si tollera in fase di collaudo dei pozzi fino a $2 \mathrm{mg} / \mathrm{l}$ di sostanze solide presenti e allo stesso modo si ha nelle norme sull'uso per il consumo umano un consiglio che indica una concentrazione della torbidità "Accettabile per i consumatori e senza variazioni anomale” (D.Lgs. 31/2001 Allegato 1, parte C).

Il ricircolo di acqua a pressione e temperature variabili dalle acque sotterranee riceventi può

anche portare a interazioni chimiche, provocando lo scioglimento o la precipitazione di minerali costituenti la matrice dell'acquifero.

Ad esempio:

- la solubilità della Calcite è maggiore a temperature più basse e diminuisce di quattro volte tra $0{ }^{\circ} \mathrm{C}$ e $50{ }^{\circ} \mathrm{C}$;

- la solubilità dei gas presenti comunemente nelle acque sotterranee $\left(\mathrm{CO}_{2}, \mathrm{O}_{2}\right)$, aumenta anche con temperature decrescenti.

$\mathrm{Nel}$ caso di $\mathrm{CO}_{2}$ ciò significa che l'acido carbonico è maggiormente presente nelle acque più fredde (per una data $\mathrm{pCO}_{2}$ ) e quindi i carbonati possono essere disciolti. 
$\mathrm{La}$ formazione di $\mathrm{CO}_{2}$ nelle acque può essere dovuta all'attività microbica, dalla quale si produce acido carbonico che è in grado di produrre bicarbonati e in parte carbonati con aumento del $\mathrm{pH}$.

Invece dove la $\mathrm{CO}_{2}$ è persa dall'acqua in conseguenza del degassaggio prodotto dalla diminuzione di temperatura o pressione si ha la precipitazione di carbonati, purché sufficientemente presenti nelle acque; questo fenomeno si verifica nelle pompe di calore a circuito aperto quando acque più fredde vengono immesse in acque più calde nel sottosuolo.

Seppure limitata alla parte dei filtri e in parte del dreno, la precipitazione determina anch'essa un intasamento rispetto alla possibilità di iniettare acque nel sottosuolo, con effetto simile a quello delle bolle di gas e del materiale in sospensione, producendo resistenza al flusso e quindi riducendo l'efficienza del pozzo.

Nella Fig. 14 è riportato il diagramma di stabilità per il Ferro nelle acque che è un metallo che frequentemente si trova nelle acque e che determina problemi all'utilizzo dei pozzi.

Nella zona di combinazione di valori di $\mathrm{pH}$-Eh si determinano condizioni per reazioni di precipitazione dei metalli che sono relativamente veloci (dell'ordine dei minuti) in acque neutre. Tuttavia questi diagrammi forniscono indicazioni di massima circa le possibili reazioni, in quanto ignorano la presenza di reazioni di e con altre sostanze organiche e inorganiche e la possibilità di formazione di complessi.

Al contrario, la dissoluzione di minerali può produrre la creazione di nuovi percorsi anche attraverso il dreno, ma non nel caso sia costituito solo da litotipi silicei.

In ogni caso all'interno della gamma di temperature previste dalle pompe di calore a bassa entalpia, l'effetto complessivo della temperatura sulla solubilità di minerali è probabilmente modesta.

Altri fattori quali il $\mathrm{pH}$ sono suscettibili di essere il controllo dominante, ma questi sono anche influenzati da variazioni di temperatura attraverso la solubilità della $\mathrm{CO}_{2}$.

Le variazioni di temperatura influiscono anche sulla mobilità delle acque in quanto viene modificata anche la permeabilità dei litotipi.

In relazione alla modifica di viscosità cinematica $v\left(\mathrm{~m}^{2} / \mathrm{s}\right)$ dell'acqua data da:

$$
v=\frac{\mu}{\rho}
$$

ad una variazione di temperatura tra 5 e $25^{\circ} \mathrm{C}$ corrisponde in una ghiaia e sabbia di un acquifero di permeabilità intrinseca $\mathrm{k}=10^{-10} \mathrm{~m}^{2}$ un aumento di conducibilità idraulica di oltre 1.6 volte.

Anche la parte biologica può influire sulle reazioni chimicofisiche in quanto la crescita di microrgamismi e alghe sulla superficie di infiltrazione può abbassare il pH e togliere $\mathrm{CO}_{2}$ inducendo precipitazione di minerali.

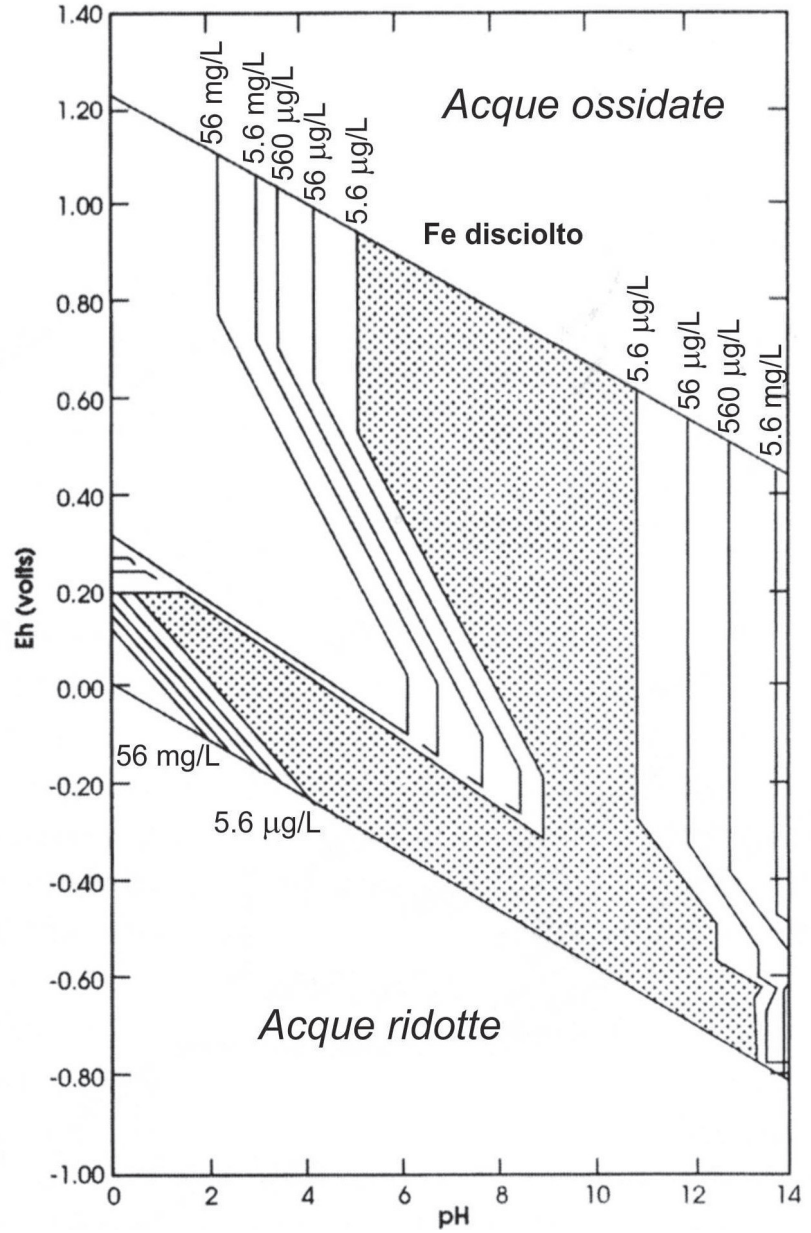

Fig. 14 - Equilibrium activity of dissolved Iron in water in relation to Eh-pH and outlined area in which it occurs the metal precipitation (Hem 1985).

Fig. 14 - Attività all'equilibrio del Ferro disciolto nelle acque in funzione di Eh-pH e zona evidenziata nella quale si verifica la precipitazione del metallo (Hem 1985). 


\section{BIBLIOGRAFIA}

Alford, G. Cullimore R. (1999) The Application of Heat and Chemicals in the Control of Biofouling Events in Wells. Lewis Publishers, Boca Raton, Florida

Ausseur J.Y., Sauty J.J. (1982) Exploitation de doublets de forage pour le chauffage ou la climatisation avec pompes à chaleur, les milieux discontinus en hydrogéologie. Documents du BRGM n. 45, Orléans

Banks D. (2009) An introduction to 'thermogeology' and the exploitation of ground source heat. Quarterly Journal of Engineering Geology and Hydrogeology, Vol. 42, 283-293.

Beretta G.P. (1984) Condizioni idrogeologiche per lo sfruttamento degli acquiferi poco profondi mediante pompe di calore "Hydrogeological conditions for the exploitation of shallow aquifers through heat pumps". Ingegneria Ambientale, vol. 13, n. 9, settembre, Milano

Bichara A.F. (1986) Clogging of recharge wells by suspended solids. J. Irrig. Drain Eng., 112(3): 210-224

Characklis, W.G., Marshall K.C. (1990) Biofilms. John Wiley and Sons, Inc, New York

Cullimore M.R. (1978) The Identification, Cultivation and Control of Iron Bacteria in Ground Water. Aquatic Microbiology, Editors Skinner \& Shewan Academic Press

Cullimore M.R. (1999) Microbiology of Well Biofouling. Lewis Publishers, CRC Press, Boca Raton, Florida

Environmental Security \& Technology Certification Program (ESTCP) (2005) A review of biofouling controls for enhanced in situ bioremediation of groundwater. ESTCP PROJECT ER-0429, 64 pp., Arlington, Virginia.

Hem J.D. (1985) Study and Interpretation of the Chemical Characteristics of Natural Water. U.S. Geological Survey Water Supply Paper 224i54, 264 pp.

Le Feuvre P., St John Cox C. (2009) Ground source heating and cooling pumps - state of play and future trends. Evidence Directorate, Environment Agency of England and Wales, ISBN 978-1-84911136-2.

Lippmann M.J., Tsang C.F. (1980) Groundwater use for cooling: associated temperature changes. Ground Water, 18(5), 452-458

Mayer C., Moritz R., Kirschner C., Borchard W., Maibaum R., Wingender J., Flemming H. (1999) The Role of Intermolecular Interactions: Studies on Model Systems for Bacterial Biofilms. Int. J. Bio. Macromol. 26: 3-16.

Olsthoorn T.N. (1982) The clogging of recharge wells, main subjects. Working group on recharge wells, Assembled within the research program of the VEWIN Association, 139 pp., Rijswijk

Pyne, R.G.D. (2005) Aquifer storage and recovery: A guide to groundwater recharge through wells. Second Edition ISBN 0-9774337090000, 609 pp.

Rahman, M. D. A., Snerdon, E. T., Hiler, E. A., (1969) Effect of Sediment Concentration on Well Recharge through Fine Sand Aquifer. Water Resources Research, Vol. 5, No. 3, June, 1969, pp. 641-646

Repubblica Italiana (2001) Attuazione della direttiva 98/83/CE relativa alla qualità delle acque destinate al consumo umano. D.Lgs. 2 febbraio 2001, n. 31, G.U. n.41/L del 3 marzo 2001, Roma
Repubblica Italiana (2006) Norme in materia ambientale. D.Lgs. 3 aprile 2006, n. 152, G.U. Serie generale, 14-4-2006, Roma

Repubblica Italiana (2009) Attuazione della direttiva 2006/118/CE, relativa alla protezione delle acque sotterranee dall'inquinamento e dal deterioramento. (09G0038). D.Lgs. 16 marzo 2009, n. 30 , G.U. n. 79 del 4 aprile 2009, Roma

Repubblica Italiana (2010) Riassetto della normativa in materia di ricerca e coltivazione delle risorse geotermiche, a norma dell'articolo 27, comma 28, della legge 23 luglio 2009, n. 99). D.Lgs. 11 febbraio 2010, n. 22, G.U. n. 45 del 24 febbraio 2010, Roma

Repubblica Italiana (2013) Conversione, con modificazioni, del decreto-legge 21 giugno 2013, n. 69 Disposizioni urgenti per il rilancio dell'economia. Legge 9 agosto 2013, n. 98, G.U. n. 194 del 20 agosto 2013, Roma

Repubblica Italiana (2016) Regolamento recante criteri per il rilascio dell'autorizzazione al ravvenamento o all'accrescimento artificiale dei corpi idrici sotterranei al fine del raggiungimento dell'obiettivo di qualità, ai sensi dell'articolo 104, comma 4-bis, del decreto legislativo 3 aprile 2006, n. 152. (16G00111). Decreto del ministero dell'Ambiente e della tutela del territorio e del mare 2 maggio 2016, n. 100, G.U. del 13 giugno 2016, n.136, Roma

Schippers J.C., Verdouw J. (1980) The Modified-Fouling Index. A Method for Determining the Fouling Characteristics of Water. Desalination 32 (1980) pp 137-148.

Schomburgk S., Goyeneche O., Gateau C., Vernoux, J., Denis L. (2005) Guide d'aide à la decision pour l'installation de pompes a chaleur sur nappe aquifere en region Ile-de-France: Partie1-Atlas hydrogeologique. BRGM, BRGM/RP-53306-FR

Smith, S.A. (1995) Monitoring and Remediation of Remediation Wells. Problem Prevention, Maintenance, and Rehabilitation. CRC Press, Lewis Publishers, Boca Raton, Florida

Sniegocki R.T. (1963) Geochemical Aspects of Artificial Recharge Water-Supply. Paper 1615-E, Washington, USGS-1963, 41 pp.

Sniegocki R.T. Reed J.E. (1963) Principles of Siphons with respect to the Artificial Recharge, Studies in the Grand-Prairie region, Arkansas Washington. USGS, 1963, Water-Supply Paper 1615-D, 1965 pp.

Stumm W. (1977) Chemical interaction in particle separation. Environmental Science and Technology, 11, $1070 \mathrm{pp}$.

Taylor, S.W., Jaffe P.R. (1991) Enhanced In-Situ Biodegradation and Aquifer Permeability Reduction. Journal of Environmental Engineering. 117(1): 25-46

Unione Europea (2000) Direttiva 2000/60/CE del Parlamento Europeo e del Consiglio del 23 ottobre 2000 che istituisce un quadro per l'azione comunitaria in materia di acque. G.U. delle Comunità europee L 327/1, 22/12/2000

Unione Europea (2006) Direttiva 2006/118/CE del Parlamento Europeo e del Consiglio del 12 dicembre 2006 sulla protezione delle acque sotterranee dall'inquinamento e dal deterioramento. G.U. dell'Unione europea L 372/19, 27/12/2006 